\title{
The Assessment of Concerns, Opinions and Perceptions of Bank Customers to find the Significant Metrics for Deployment of Biometrics in E-Banking
}

\author{
Munish Sabharwal, PhD \\ Executive Director, \\ KITE Group of Institutions, \\ Meerut (U.P.) INDIA
}

\begin{abstract}
The research work was conducted with the objective to find the significant metrics for biometrics deployment in e-banking through an assessment of the concerns, opinions and perceptions of bank customers regarding the implementation of biometrics in e-banking. This paper is pursued by collecting information through survey of customers the branches of scheduled banks from the area of research chosen by researcher.The researcher has used probability stratified sampling and scheduled banks categorized by RBI have been divided into groups referred to as strata on the basis of the total turnover, then the banks are selected from the various groups using random sampling and finally a branch of the selected bank with the one of highest turnover is randomly selected and the customers of the branch are chosen using non-probability convenience sampling. The study suggests that the overall significant metrics for the deployment of Biometric technology in E-Banking with the bank customer's perspective areReliability, Performance, Circumvention Resistance, Ergonomics, Minimum User Participation, Privacy Issues, Health Concerns, Data Security and Trust.
\end{abstract}

\section{Keywords}

Biometric Technologies,Biometrics Deployment, Bank Customer Perception, E-Banking, Significant Metrics for Biometrics Implementation

\section{INTRODUCTION}

In the modern era the data is in digital or electronic form and its security and privacy has become very fundamental and the issues concerning the security and privacy of electronic data have remained a challenge for years.. Personal identification (authentication) plays a vital role in contemporary society (Stanley et al., $2009^{1}$ ) as it can help in attaining the security by the identification of a person.

For the last two decades information technology has been broadly using Passwords or Personal Identification Numbers (PIN's) to validate a user to a system though the recognition of a PIN does not, however, imply the detection of the person's identity because if anyone acquires access to a PIN, a card or any other such 'key' that is being used to get entrée to a system or device then that person can breach the security. This implies that systems that are reliant on high access security cannot always rely on these kinds of 'Keys', since they cannot ensure the identity of a user is who s/he claims to be. Biometrics could be used to obtain trust to a device instead of PIN's or passwords.

Authentication can be performed in three different ways, firstly based on something you know (passwords and Pin's), secondly based on something you have (ID card or Token) and lastly based on something you are (biometrics). The first two methods are standard methods of personal identification i.e. something you know and something you have (passwords and tokens) and they have had their limitations i.e. One does not remember what he/she knew and one does not have what one had (Russell Kay, 2009 ${ }^{2}$; Boatwright, 2007 ${ }^{3}$ ). The limitations linked with the conventional methods of authentication can be shielded using a new authentication mechanism-based on what you are (biometrics) was introduced. Also now with the wide use of IT and Gadgets one has to remember passwords for net banking, personal and professional emails, government and organizational login, social networking sites, Mobile Banking, Cloud Storages, EStores and other related sites, with the need to remembering one additional password virtually every few days. Remembering all such passwords is getting increasingly cumbersome and difficult as well forgetting them involves hassles and disclosure or leak may prove to be fatal. Therefore a novel, convenient as well as secure technology is required for authentication as well as transaction operation.

The controversy regarding biometric technology has been about invading the privacy of individuals and the doubts and concerns that they about their privacy. The critics of biometrics assertthat biometrics is a threat to the individual's privacy. Though biometrics has been put into practice in many organizations but still there is a long way to go. It is very important that the responses, both perceived and behavioral, of the citizens and end users are considered when deigning and deploying system having digital identities (Dwivedi, et al., $2003^{4}$ ).

Chandra and Calderon $\left(2005^{5}\right)$ explain the user's issues involved in the deployment of biometrics technology. They elaborate that if the people concerns i.e. trust, user acceptance, privacy concerns are not addressed then there is a potential threat to system failure. It would be surprising to deploy biometrics technology without measuring the people's perceptions about biometrics technology. Using biometrics raises concerns about the public's perception of a possible intrusion of their privacy. One can generally say that the less intrusive the biometric, the more likely it is that it will be accepted by the users.

Since the use of biometric technologies involves sharing of an individual's indispensable identity data, the opinions of users and bankers before their deployment must essentially be considered. 


\section{REVIEW OF LITERATURE}

Since the use of biometric technologies involves sharing of an individual's indispensable identity data, the opinions of users and bankers before their deployment must essentially be considered. According to Woodward $(1997)^{6}$ there are two different blogs of opinions about biometrics as it's a relatively new technology. The critics claims it as a privacy invader while the pro biometrics blog details and supports the biometrics technology for improved security and the greater services. People have concerns regarding their security and privacy when dealing with biometrics. In this paper the question (Is biometrics a privacy friend or privacy foe?) is answered by first explaining the biometrics technology and itsexpected uses in daily life. The privacy aspects of biometrics are analyzed from invader as well as protective perspective of biometrics. Though people have concerns regarding the biometrics but when analyzed, the biometrics was found to be a technology that improves the privacy as well as the security of the users.

${ }^{7}$ Wayne Penny in SANS Institute in 2002 suggests that two of the issues to be overcome with biometric systems and public acceptance are communications, by the vendors or implementers, and public perception of the technology. The individual must be able to understand the behaviour of the system to assess its capabilities to protect information and function in an open and secure manner.

Salil Prabhakar et. al $\left(2003^{8}\right)$ in their study suggest thatthe use of biometrics indeed raises several privacy concerns. A sound trade-off between security and privacy might be necessary; but one can only enforce collective accountability and acceptability standards through common legislation. On the positive side of the privacy issue, biometrics provides tools to enforce accountable logs of system transactions and to protect individual's right to privacy.

Chandra and Calderon $(2005)^{9}$ discussed the challenges and difficulties that biometrics technology face in becoming the core technology for authentication in information systems. Different types of challenges and issues i.e. business issues, operational issues and the people issues are studied. There is a need to approach these challenges in a way that satisfies the user concerns.

Elliot et al. (2007) ${ }^{10}$ used the survey methodology in order to understand and analyze the citizen's perceptions, opinions and concerns of biometrics technology. The issues like security, safety and privacy concerns were asked in the survey. The results mentions that the people were pro biometrics i.e. they agreed that biometrics usage will enhance security but most of the respondents had concerns about the their privacy (who will use that data and how it is made sure that only the authentic people use that data). People seemed welcoming to biometrics technology but also they had safety concerns from using biometrics technology i.e. iris and scan technology. In short the people were willing to use biometrics technology but there was a certain lack of trust with some governmental institutes. There is also a need to educate people about the biometrics as most of the concerns can be removed if proper guidance and education is delivered about biometrics technology.

The research work of Furnell and Evangelatos (2007) ${ }^{11}$ also explains the people perceptions about biometrics technology. The survey conducted by the researchers revealed that there is a certain level of acceptance towards biometrics among people. Moreover the survey states that TV, Newspapers and
Internet are the sources that people get information of biometrics technology from. People were found having concerns of health risks while using biometrics devices. Privacy concerns, like who will access the stored data and how it will be used, were noticeable among people. They survey summarize that although there is an adequate user acceptance for biometrics with certain concerns, there is a need to take steps for the awareness of people about their perceived concerns.

Andrew Patrick $\left(2007^{12}\right)$ in his essay "Biometrics and Identity Theft" quotes that Research studies have found that users' concerns about biometric misuse and privacy invasions are large and poorly articulated. Potential users are also concerned about the reliability of new technology. Moreover, research has shown that users find biometrics systems to be less hygienic and more stressful than traditional PIN systems. Some groups have also expressed concerns about health risks caused by biometric systems, such as eye damage caused by the near infrared illumination used for iris scanning. Users also reported significant fears that criminals may do them harm to obtain the biometric (e.g., cut off their finger).

Michael Colin Breward $\left(2009^{13}\right)$ in his study to determine the Factors Influencing Consumer Attitudes towards Biometric Identity Authentication Technology within the Canadian Banking Industry suggests that the contextual factors of privacy and security concerns and usefulness have a bigger impact upon attitude as compared to innate personality traits. In addition, while voluntariness appears to have no effect, control has a significant impact upon attitude as well as privacy and security concerns and usefulness.

Antonio Pooe and Les Labuschagne $\left(2010^{14}\right)$ investigate the cause for the slow adoption of biometric authentication in the South African (SA) banking sector and they suggest that several bank employees have been exposed to biometric technology before. Though some appear tohave never used this technology before, this does not affect their opinion on biometrics as a possible alternative to current security challenges in the banking sector. They also established that there is a definite interest in the use of biometric technology across different banking channels and their findings showed that the participating local banks have and are investigating biometric authentication and that these investigations were not limited to any particular biometric trait and their study highlights the problem areas affecting biometric adoption.

A. Pooe $(2011)^{15}$ in his study aimed to identify factors impacting on the adoption of biometric authentication in the South African banking sector as a means of authentication. The study constitutes exploratory research and is limited to the use of biometric technology within the financial services sector. Within this sector, specific focus is placed on the four leading South African banks. A survey was conducted, and the findings show common agreement and acceptance of biometric authentication as a way to improve information security in the various banking channels despite its not being widely implemented. With regard to factors influencing the adoption of biometric authentication, the study identified three main adoption inhibitors. This study contributes to the greater body of knowledge on the use of biometrics for banking applications by providing insight into current practices and perceptions.

Seyyede Samine Hosseini and Dr. Shahriar Mohammadi(2012 ${ }^{16}$ ) in their study investigated the employees' and customers' conceptualizations about the introduction and accomplishment of a biometric 
authentication system in Saman bank of Shiraz, Iran and concluded that although the participants are aware of biometric technology's benefits, they believe that cultural and economic problems could be the two obstacles for implementation of such an authentication system in the banks of Iran.

Dhurgham T. Ahmad and Mohammad Hariri $\left(2012^{17}\right)$ in their study suggest that the major determinants which relate to the intention to adopt the mentioned technology are perceived usefulness and the ease of use. Apart from this the concept of Self efficacy was also identified as a contributing factor in order to accept the technology. Therefore it is very clear according to them that the perception of users towards the adoption of biometrics in e-banking is directly influenced by the attitude of users and their intention to make use of the technology.

Sookeun Byun and Sang-Eun Byun $\left(2013^{18}\right)$ in their study investigate multiple aspects of the benefits and risks that consumers perceive in using biometric technology. A survey was conducted by contacting the actual customers of an American bank that has utilized fingerprint technology at its ATMs. Banks thus may highlight intrinsic values, such as the novelty of biometrics, to motivate the use of the technology. However, to promote potential users' adoption decisions, banks need to educate them about the security benefits of financial transactions under the technology. The result also showed that the current users were highly concerned about information privacy risk in using the fingerprint ATMs. Therefore, banks are advised to develop internal policies to protect personal biometric data from any identity theft or illegal uses to encourage continuous usage by the current users.

Adewale Adeyinka A. et. al. $\left(2014{ }^{19}\right)$ in their empirical evaluation capture the factors influencing the perception of the bank management and users. The analysis of the survey of 740 respondents cutting across different age groups and educational backgrounds showed that management and customers of strongly support the adoption of biometric ATM in Nigeria.

There have been several studies that have assessed the concerns, opinions and perceptions of Bank employees as well as bank customers regarding use of biometrics in banking, the research gap is that none of the studies have specifically aimed at finding significant metrics for deployment of biometric in e-banking by assessing the concerns, opinions and perceptions of bank customers.

\section{OBJECTIVES OF THE STUDY}

The research work was conducted with the following objective:

- To assess the concerns, opinions and perceptions of bank customers regarding the implementation of biometrics in e-banking

- To find the significant metrics for biometrics deployment in e-banking

\section{RESEARCH METHODOLOGY}

Survey method is used to identify and analyze the concerns, opinions and perceptions of customers regarding the deployment of biometric technology in e-banking. To conduct the survey of customers, the researcher has focused his research only on the scheduled banks in India. The area of research chosen by the researcher is Meerut city. In order to get a sample that is a representative of the whole population the researcher has used probability stratified sampling and scheduled banks categorized by RBI have been divided into groups referred to as strata on the basis of the total turnover, then the banks are selected from the various groups or strata using random sampling and finally a branch of the selected bank with the one of highest turnover in Meerut is randomly selected. The customers of the branch are chosen using nonprobability convenience sampling. The details of the survey methodology used in the research are as given below:

\subsection{Population}

The list of scheduled banks is as given in the Table below:

Table 1. List Of Scheduled Banks (Bank Groupwise)

\begin{tabular}{|c|c|c|c|}
\hline S.No & Bank Name & $\begin{array}{c}\text { S.N } \\
\text { o }\end{array}$ & Bank Name \\
\hline \multirow[t]{2}{*}{1} & SBI \& its Associates & 4 & Kotak Mahindra Bank \\
\hline & Nationalized Banks & 5 & IndusInd Bank \\
\hline 1 & $\begin{array}{l}\text { Punjab National } \\
\text { Bank }\end{array}$ & 6 & Yes Bank \\
\hline 2 & Central Bank of India & 7 & $\begin{array}{l}\text { Development } \quad \text { Credit } \\
\text { Bank }\end{array}$ \\
\hline 3 & Bank of Baroda & & FOREIGN BANKS \\
\hline 4 & Bank of India & 1 & Standard Chartered Bank \\
\hline 5 & Canara Bank & 2 & HSBC \\
\hline 6 & Union Bank of India & 3 & Citibank \\
\hline 7 & Syndicate Bank & 4 & $\begin{array}{l}\text { The Royal Bank of } \\
\text { Scotland }\end{array}$ \\
\hline 8 & Allahabad Bank & 5 & Deutsche Bank \\
\hline 9 & UCO Bank & 6 & DBS Bank \\
\hline 10 & $\begin{array}{l}\text { Indian } \quad \text { Overseas } \\
\text { Bank }\end{array}$ & 7 & Barclays Bank \\
\hline 11 & Indian Bank & 8 & BNP Paribas \\
\hline 12 & $\begin{array}{l}\text { Oriental Bank of } \\
\text { Commerce }\end{array}$ & 9 & Credit Agricole Bank \\
\hline 13 & Andhra Bank & 10 & Bank of America \\
\hline 14 & United Bank of India & 11 & Bank of Nova Scotia \\
\hline 15 & Bank of Maharashtra & 12 & Shinhan Bank \\
\hline 16 & Corporation Bank & 13 & State Bank of Mauritius \\
\hline 17 & Dena Bank & 14 & $\begin{array}{l}\text { The Bank of Tokyo- } \\
\text { Mitsubishi UFJ }\end{array}$ \\
\hline 18 & Vijaya Bank & 15 & $\begin{array}{l}\text { Abu Dhabi Commercial } \\
\text { Bank }\end{array}$ \\
\hline \multirow[t]{2}{*}{19} & Punjab \& Sind Bank & 16 & $\begin{array}{l}\text { Bank of Bahrain \& } \\
\text { Kuwait }\end{array}$ \\
\hline & $\begin{array}{l}\text { Other Public Sector } \\
\text { Banks }\end{array}$ & 17 & Mashreq Bank \\
\hline \multirow[t]{2}{*}{1} & IDBI Bank Limited & 18 & Mizuho Corporate Bank \\
\hline & $\begin{array}{l}\text { Old Private Sector } \\
\text { Banks }\end{array}$ & 19 & $\begin{array}{l}\text { Oman International } \\
\text { Bank }\end{array}$ \\
\hline 1 & Federal Bank & 20 & Societe Generale \\
\hline 2 & South Indian Bank & 21 & Sonali Bank \\
\hline 3 & ING Vysya Bank & 22 & AB Bank \\
\hline 4 & $\begin{array}{l}\text { Jammu \& Kashmir } \\
\text { Bank }\end{array}$ & 23 & $\begin{array}{l}\text { American Express } \\
\text { Banking Corp. }\end{array}$ \\
\hline 5 & Karnataka Bank & 24 & Antwerp Diamond Bank \\
\hline 6 & Karur Vysya Bank & 25 & $\begin{array}{ll}\text { Bank } & \text { Internasional } \\
\text { Indonesia } & \end{array}$ \\
\hline 7 & Catholic Syrian Bank & 26 & Bank of Ceylon \\
\hline 8 & $\begin{array}{l}\text { The Dhanalaxmi } \\
\text { Bank }\end{array}$ & 27 & $\begin{array}{l}\text { Chinatrust Commercial } \\
\text { Bank }\end{array}$ \\
\hline 9 & Lakshmi Vilas Bank & 28 & $\begin{array}{l}\text { Commonwealth Bank of } \\
\text { Australia }\end{array}$ \\
\hline 10 & City Union Bank & 29 & Credit Suisse AG \\
\hline 11 & $\begin{array}{l}\text { Tamilnad Mercantile } \\
\text { Bank }\end{array}$ & 30 & FirstRand Bank \\
\hline
\end{tabular}




\begin{tabular}{|c|l|c|l|}
\hline 12 & Nainital Bank & 31 & JP Morgan Chase Bank \\
\hline 13 & Ratnakar Bank & 32 & JSC VTB Bank \\
\hline 14 & $\begin{array}{l}\text { SBI Commercial \& } \\
\text { Intl. Bank }\end{array}$ & 33 & Krung Thai Bank \\
\hline & $\begin{array}{l}\text { NEW PRIVATE } \\
\text { SECTOR BANKS }\end{array}$ & 34 & Sberbank \\
\hline 1 & ICICI Bank & 35 & UBS AG \\
\hline
\end{tabular}

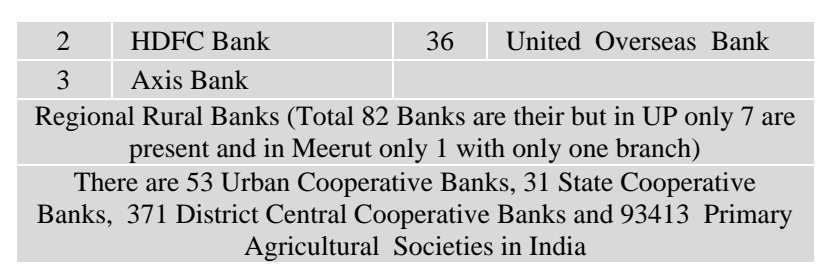

\subsection{Sample Design:}

Since the population size is very big it was not feasible to study the entire population, so the researcher decided to go for a sample survey

${ }^{20}$ Table 1.01: List Of Public Sector Banks With Total Turnover And Profitsbi \& Its Associates

\begin{tabular}{|c|c|c|c|c|c|c|}
\hline BANK & $\begin{array}{l}\text { TOTAL } \\
\text { BRANCHE } \\
\mathrm{S} \text { as on 31st } \\
\text { March 2011 }\end{array}$ & $\begin{array}{l}\text { Total No. of } \\
\text { Employees as } \\
\text { on 31st March } \\
2011\end{array}$ & $\begin{array}{l}\text { Business per } \\
\text { employee on } \\
31 \text { st March } \\
2011\end{array}$ & $\begin{array}{lr}\text { Profit } & \text { per } \\
\text { Employee on } \\
31 \text { st March } \\
2011\end{array}$ & $\begin{array}{l}\text { Total Turnover } \\
\text { on 31st March } \\
2011\end{array}$ & $\begin{array}{lr}\text { Total Profit } \\
\text { on } \\
\text { March } & 2011 \text { st }\end{array}$ \\
\hline SBI \& its Associates & 17,913 & 282453 & 793.06 & 4.2 & 224002176.2 & 1186302.6 \\
\hline \multicolumn{7}{|c|}{ NATIONALIZED BANKS } \\
\hline BANK & $\begin{array}{l}\text { TOTAL } \\
\text { BRANCHE } \\
\text { S as on } 31 \text { st } \\
\text { March } 2011\end{array}$ & $\begin{array}{l}\text { Total No. of } \\
\text { Employees as } \\
\text { on 31st March } \\
2011\end{array}$ & $\begin{array}{l}\text { Business per } \\
\text { employee on } \\
31 \text { st March } \\
2011\end{array}$ & $\begin{array}{lr}\text { Profit } & \text { per } \\
\text { Employee on } \\
31 \mathrm{st} \text { March } \\
2011\end{array}$ & $\begin{array}{l}\text { Total Turnover } \\
\text { on 31st March } \\
2011\end{array}$ & $\begin{array}{lr}\text { Total } & \text { Profit } \\
\text { on } & 31 \text { st } \\
\text { March } & 2011\end{array}$ \\
\hline Punjab National Bank & 4,855 & 56963 & 1017.8 & 8.35 & 57976941.4 & 475641.05 \\
\hline Canara Bank & 3,737 & 34015 & 835.17 & 3.96 & 28408307.55 & 134699.4 \\
\hline Bank of India & 3,352 & 39385 & 1333 & 11 & 52500205 & 433235 \\
\hline Bank of Baroda & 3,303 & 39785 & 1284 & 6.2 & 51083940 & 246667 \\
\hline Central Bank of India & 3,252 & 43397 & 1228.18 & 9.76 & 53299327.46 & 423554.72 \\
\hline Union Bank of India & 3,051 & 29462 & 1043 & 7.5 & 30728866 & 220965 \\
\hline Syndicate Bank & 2,491 & 26288 & 875.44 & 3.99 & 23013566.72 & 104889.12 \\
\hline Indian Overseas Bank & 2,373 & 21227 & 1063 & 6.7 & 22564301 & 142220.9 \\
\hline UCO Bank & 2,192 & 23046 & 1069 & 4.19 & 24636174 & 96562.74 \\
\hline Allahabad Bank & 2,167 & 25626 & 1005 & 4.16 & 25754130 & 106604.16 \\
\hline Indian Bank & 1,829 & 19311 & 930 & 8.88 & 59230 & 171481.68 \\
\hline $\begin{array}{lll}\text { Oriental Bank of } \\
\text { Comm. }\end{array}$ & 1,640 & 16618 & 1419.5 & 9.04 & 23589251 & 150226.72 \\
\hline United Bank of India & 1,603 & 14098 & 1165 & 9 & 16424170 & 126882 \\
\hline Andhra Bank & 1,556 & 15062 & 860 & 3.48 & 12953320 & 52415.76 \\
\hline Bank of Maharashtra & 1,505 & 13861 & 825 & 2.38 & 11435325 & 32989.18 \\
\hline Corporation Bank & 1,268 & 13861 & 1572.79 & 10.92 & 21800442.19 & 151362.12 \\
\hline Vijaya Bank & 1,191 & 9953 & 1077 & 6.15 & 9381 & 61210.95 \\
\hline Dena Bank & 1,186 & 11415 & 928 & 6.3 & 10593120 & 71914.5 \\
\hline Punjab \& Sind Bank & 941 & 8107 & 1190 & 6 & 9647330 & 48642 \\
\hline \multicolumn{7}{|c|}{ OTHER PUBLIC SECTOR BANKS } \\
\hline BANK & $\begin{array}{l}\text { TOTAL } \\
\text { BRANCHE } \\
\text { S as on 31st } \\
\text { March } 2011\end{array}$ & $\begin{array}{l}\text { Total No. of } \\
\text { Employees as } \\
\text { on 31st March } \\
2011\end{array}$ & $\begin{array}{lr}\text { Business } & \text { per } \\
\text { employee on } \\
31 \text { st March } \\
2011\end{array}$ & $\begin{array}{lr}\text { Profit } & \text { per } \\
\text { Employee on } \\
31 \text { st March } \\
2011\end{array}$ & $\begin{array}{l}\text { Total Turnover } \\
\text { on 31st March } \\
2011\end{array}$ & $\begin{array}{lr}\text { Total Profit } \\
\text { on } & 31 \text { st } \\
\text { March } & 2011\end{array}$ \\
\hline IDBI Bank Ltd. & 806 & 13602 & 2346.41 & 11.93 & 31915868.82 & 162271.86 \\
\hline
\end{tabular}

${ }^{21}$ Table 1.02: List Of Private Sector Banks With Total Turnover And Profit

\begin{tabular}{|c|c|c|c|c|c|c|}
\hline \multicolumn{7}{|c|}{ OLD PRIVATE SECTOR BANKS } \\
\hline BANK & $\begin{array}{l}\text { TOTAL } \\
\text { BRANCHE } \\
\mathrm{S} \text { as on } 31 \mathrm{st} \\
\text { March } 2011\end{array}$ & $\begin{array}{l}\text { Total No. of } \\
\text { Employees as } \\
\text { on 31st March } \\
2011\end{array}$ & $\begin{array}{l}\text { Business per } \\
\text { employee on } \\
31 \text { st March } \\
2011\end{array}$ & $\begin{array}{l}\text { Profit per } \\
\text { Employee on } \\
31 \text { st March } \\
2011\end{array}$ & $\begin{array}{l}\text { Total Turnover } \\
\text { on 31st March } \\
2011\end{array}$ & $\begin{array}{lr}\text { Total } & \text { Profit } \\
\text { on } & 31 \text { st } \\
\text { March } & 2011\end{array}$ \\
\hline Jammu \& Kashmir Bank & 632 & 5357 & 918 & 5 & 4917726 & 26785 \\
\hline ING Vysya Bank & 504 & 6909 & 674.79 & 4.53 & 4662124.11 & 31297.77 \\
\hline Karnataka Bank & 503 & 7938 & 856 & 8 & 6794928 & 63504 \\
\hline South Indian Bank & 483 & 5795 & 771 & 4 & 4467945 & 23180 \\
\hline Karur Vysya Bank & 369 & 4574 & 935 & 9.09 & 4276690 & 41577.66 \\
\hline Dhanlaxmi Bank & 360 & 2820 & 537 & 0.45 & 1514340 & 1269 \\
\hline City Union Bank & 273 & 3665 & 589.22 & 0.71 & 2159491.3 & 2602.15 \\
\hline
\end{tabular}




\begin{tabular}{|c|c|c|c|c|c|c|}
\hline Catholic Syrian Bank & 269 & 2626 & 719 & 3.85 & 1888094 & 10110.1 \\
\hline Lakshmi Vilas Bank & 248 & 2836 & 781 & 8 & 2214916 & 22688 \\
\hline $\begin{array}{l}\text { Tamilnad Mercantile } \\
\text { Bank }\end{array}$ & 232 & 2531 & 959.18 & 9.91 & 2427684.58 & 25082.21 \\
\hline Ratnakar Bank & 101 & 725 & 585 & 6 & 424125 & 4350 \\
\hline Nainital Bank & 100 & 907 & 435 & 1 & 394545 & 907 \\
\hline $\begin{array}{l}\text { SBI Comm. \& Intl. } \\
\text { Bank }\end{array}$ & 2 & 72 & 955.05 & 5.85 & 68763.6 & 421.2 \\
\hline \multicolumn{7}{|c|}{ NEW PRIVATE SECTOR BANKS } \\
\hline BANK & $\begin{array}{l}\text { TOTAL } \\
\text { BRANCHE } \\
\mathrm{S} \text { as on } 31 \mathrm{st} \\
\text { March } 2011\end{array}$ & $\begin{array}{l}\text { Total No. of } \\
\text { Employees as } \\
\text { on 31st March } \\
2011\end{array}$ & $\begin{array}{lr}\text { Business } & \text { per } \\
\text { employee } & \text { on } \\
31 \text { st March } & \text { Ma11 }\end{array}$ & $\begin{array}{lr}\text { Profit } & \text { per } \\
\text { Employee on } \\
31 \text { st March } \\
2011\end{array}$ & $\begin{array}{l}\text { Total Turnover } \\
\text { on } 31 \text { st March } \\
2011\end{array}$ & $\begin{array}{lr}\text { Total Profit } \\
\text { on } & 31 \text { st } \\
\text { March } & 2011\end{array}$ \\
\hline HDFC Bank & 1963 & 55752 & 653 & 7.37 & 36406056 & 410892.24 \\
\hline Axis Bank & 1377 & 26435 & 1366 & 14 & 36110210 & 370090 \\
\hline Kotak Mahindra Bank & 322 & 11337 & 535 & 8 & 6065295 & 90696 \\
\hline IndusInd Bank & 303 & 7008 & 843.98 & 8.24 & 5914611.84 & 57745.92 \\
\hline Yes Bank & 215 & 3929 & 2220.25 & 20.89 & 8723362.25 & 82076.81 \\
\hline $\begin{array}{ll}\text { Development } & \text { Credit } \\
\text { Bank } & \\
\end{array}$ & 82 & 2174 & 506 & 1 & 1100044 & 2174 \\
\hline
\end{tabular}

Table 1.03: Details Of Foreign, Regional Rural, Urban Cooperative And Rural Cooperative Banks

\begin{tabular}{|l|l|}
\hline Foreign Banks & $\begin{array}{l}\text { The foreign banks have been excluded by the researcher as most of them are single or less } \\
\text { than three branch banks in vast country like India and their presence is limited to mega- } \\
\text { metros only, also the research area of researcher is Meerut City which does not have any } \\
\text { foreign bank branch. }\end{array}$ \\
\hline Regional Rural Banks & $\begin{array}{l}\text { REGIONAL RURAL BANKS (Total 82 Banks are there but in UP only 7 are present and } \\
\text { in Meerut only one bank - Sarva UP Gramin has presence with only one branch }\end{array}$ \\
\hline Urban Cooperative Banks & $\begin{array}{l}\text { The Urban Cooperative Banks have been excluded by the researcher as most of the } \\
\text { branches of the bank are limited to three cities in a vast country like India and also the } \\
\text { research area of researcher is Meerut City which does not have any Urban Cooperative } \\
\text { Bank branch. }\end{array}$ \\
\hline Rural Cooperative Banks & Zila Sahkari Bank, Meerut \\
\hline
\end{tabular}

The various strata divided by using stratified sampling and the randomly selected banks from each stratum are as listed below:

\begin{tabular}{|c|c|c|}
\hline \multicolumn{2}{|l|}{ SBI \& Associates } & SBI \\
\hline \multicolumn{3}{|l|}{ Nationalized Banks } \\
\hline $\begin{array}{ll}\text { Total } & \text { Turnover } \\
\text { Range } & \\
\end{array}$ & $\begin{array}{c}\text { Banks within the } \\
\text { Range }\end{array}$ & $\begin{array}{c}\text { Selected } \\
\text { Bank }\end{array}$ \\
\hline$<30000000$ & $\begin{array}{c}\text { PNB, Canara Bank, } \\
\text { BOB, BOI }\end{array}$ & PNB \\
\hline $\begin{array}{l}30000000- \\
25000000\end{array}$ & $\begin{array}{l}\text { Union Bank of India, } \\
\text { Central Bank of India, } \\
\text { IOB, UCO Bank, }\end{array}$ & $\begin{array}{c}\text { Central } \\
\text { Bank }\end{array}$ \\
\hline $\begin{array}{l}25000000- \\
20000000\end{array}$ & $\begin{array}{c}\text { OBC, Syndicate Bank, } \\
\text { Allahabad Bank }\end{array}$ & $\begin{array}{l}\text { Syndica } \\
\text { te Bank }\end{array}$ \\
\hline $\begin{array}{c}20000000- \\
10000000\end{array}$ & $\begin{array}{c}\text { Corporation Bank, } \\
\text { Indian Bank, Andhra } \\
\text { Bank, United Bank of } \\
\text { India, Bank of } \\
\text { Maharashtra }\end{array}$ & $\begin{array}{c}\text { Andhra } \\
\text { Bank }\end{array}$ \\
\hline$>10000000$ & $\begin{array}{c}\text { Dena Bank, Vijaya } \\
\text { Bank, Punjab \& Sind } \\
\text { Bank }\end{array}$ & $\begin{array}{c}\text { Punjab } \\
\text { \& Sind } \\
\text { Bank }\end{array}$ \\
\hline \multicolumn{2}{|c|}{ Other Public Sector Banks } & $\begin{array}{l}\text { IDBI } \\
\text { Bank }\end{array}$ \\
\hline
\end{tabular}

\begin{tabular}{|c|c|c|}
\hline \multicolumn{3}{|c|}{ Old Private Sector Banks } \\
\hline $\begin{array}{c}\text { Total Turnover } \\
\text { Range }\end{array}$ & $\begin{array}{c}\text { Banks within the } \\
\text { Range }\end{array}$ & $\begin{array}{c}\text { Selected } \\
\text { Bank }\end{array}$ \\
\hline $8000000-6000000$ & $\begin{array}{l}\text { Federal Bank, Jammu } \\
\text { \& Kashmir Bank }\end{array}$ & $\begin{array}{c}\text { Federal } \\
\text { Bank }\end{array}$ \\
\hline $6000000-4000000$ & $\begin{array}{l}\text { South Indian Bank, } \\
\text { ING Vysya Bank, } \\
\text { Karnataka Bank, } \\
\text { Karur Vysya Bank }\end{array}$ & $\begin{array}{c}\text { South } \\
\text { Indian } \\
\text { Bank }\end{array}$ \\
\hline$<4000000$ & $\begin{array}{c}\text { Tamilnad Mercantile } \\
\text { Bank, City Union } \\
\text { Bank, Dhanlaxmi } \\
\text { Bank, Lakshmi Vilas } \\
\text { Bank, Catholic Syrian } \\
\text { Bank, Nainital Bank, } \\
\text { Ratnakar Bank, SBI } \\
\text { Comm. \& Intl. Bank }\end{array}$ & $\begin{array}{c}\text { Nainital } \\
\text { Bank }\end{array}$ \\
\hline \multicolumn{3}{|c|}{ New Private Sector Banks } \\
\hline $\begin{array}{l}\text { Total Turnover } \\
\text { Range }\end{array}$ & $\begin{array}{c}\text { Banks within the } \\
\text { Range }\end{array}$ & $\begin{array}{l}\text { Selected } \\
\text { Bank }\end{array}$ \\
\hline$<40000000$ & ICICI Bank & $\begin{array}{l}\text { ICICI } \\
\text { Bank }\end{array}$ \\
\hline
\end{tabular}




\begin{tabular}{|c|c|l|}
\cline { 3 - 3 } $40000000<35000000$ & HDFC Bank & \multicolumn{1}{|c|}{$\begin{array}{c}\text { HDFC } \\
\text { Bank }\end{array}$} \\
\hline $35000000<10000000$ & Axis Bank & Axis Bank \\
\hline \multirow{2}{*}{$>10000000$} & $\begin{array}{c}\text { Yes Bank, Kotak } \\
\text { Mahindra Bank, } \\
\text { IndusInd Bank, } \\
\text { Development } \\
\text { Credit Bank }\end{array}$ & Yes Bank \\
\hline Regional Rural Banks & & $\begin{array}{l}\text { Sarva UP } \\
\text { Gramin } \\
\text { Bank }\end{array}$ \\
\hline Rural Cooperative Banks & $\begin{array}{l}\text { Zila } \\
\text { Sahkari } \\
\text { Bank }\end{array}$ \\
\hline
\end{tabular}

Scope of Research: Since all banks follow the norms of the RBI and the technology implementation by banks is done as per the recommendations of committees formed by the Central Bank from time to time, therefore their policy for implementation of the any new technology in branches of a particular bank are same everywhere. Therefore, the area of research chosen by the researcher is Meerut city, as it is a well developed city having branches of most of the banks.

Sample Size: The list of selected banks and their branches are as shown in the table below:

\section{Table 1.04}

\begin{tabular}{|c|c|c|c|}
\hline \multicolumn{2}{|c|}{ List of banks selected } & \multicolumn{2}{|c|}{$\begin{array}{c}\text { List of branches of the selected } \\
\text { banks }\end{array}$} \\
\hline S. No. & Bank & S. & Bank Branch \\
\hline 1 & $\begin{array}{l}\text { SBI- State Bank } \\
\text { Of India }\end{array}$ & 1 & $\begin{array}{lrl}\text { SBI, } & \text { Meerut } & \text { Cantt } \\
\text { Branch, } & \text { Meerut } & \end{array}$ \\
\hline 2 & $\begin{array}{l}\text { PNB- } \\
\text { National Bank }\end{array}$ & 2 & $\begin{array}{l}\text { PNB, Sports Complex } \\
\text { Branch, Meerut }\end{array}$ \\
\hline 3 & $\begin{array}{l}\text { CBI-Central Bank } \\
\text { of Indian }\end{array}$ & 3 & $\begin{array}{l}\text { Central Bank of India, } \\
\text { Roadways Branch, } \\
\text { Meerut }\end{array}$ \\
\hline 4 & Syndicate Bank & 4 & $\begin{array}{lr}\text { Syndicate } & \text { Bank, } \\
\text { Mohkampur } & \text { Branch, } \\
\text { Meerut } & \\
\end{array}$ \\
\hline 5 & Andhra Bank & 5 & $\begin{array}{l}\text { Andhra Bank, Begum } \\
\text { Bridge Branch, Meerut }\end{array}$ \\
\hline 6 & Punjab \& Sind Bank & 6 & $\begin{array}{l}\text { Punjab \& Sind Bank, } \\
\text { Samarth Palace Branch, } \\
\text { Meerut }\end{array}$ \\
\hline 7 & IBDI Bank & 7 & $\begin{array}{l}\text { IDBI } \\
\text { Branch, }\end{array}$ \\
\hline 8 & Federal Bank & 8 & $\begin{array}{l}\text { Federal Bank, Chippi } \\
\text { Tank Branch, Meerut }\end{array}$ \\
\hline 9 & South Indian Bank & 9 & $\begin{array}{l}\text { South Indian } \\
\text { Branch, }\end{array}$ \\
\hline 10 & Nainital Bank & 10 & $\begin{array}{l}\text { Nainital Bank, Meerut } \\
\text { Branch, Meerut }\end{array}$ \\
\hline 11 & ICICI Bank & 11 & \begin{tabular}{lcc|} 
ICICI & Bank, & Rajlok \\
Branch, & Meerut & \\
\end{tabular} \\
\hline 12 & HDFC Bank & 12 & $\begin{array}{l}\text { HDFC } \\
\text { Palace }\end{array}$ \\
\hline 13 & Axis Bank & 13 & $\begin{array}{l}\text { Axis Bank, Boundary } \\
\text { Road Branch, Meerut }\end{array}$ \\
\hline 14 & Yes Bank & 14 & \begin{tabular}{lll|} 
Yes & Bank, & Meerut \\
Branch, & Meerut & \\
\end{tabular} \\
\hline 15 & $\begin{array}{lll}\text { Sarva } & \text { UP } & \text { Gramin } \\
\text { Bank } & & \end{array}$ & 15 & $\begin{array}{l}\text { Sarva UP Gramin Bank, } \\
\text { Madhav Puram Branch, }\end{array}$ \\
\hline
\end{tabular}

\begin{tabular}{||l||l||lr||}
\hline \hline & \multicolumn{1}{|c||l||}{} & & Meerut \\
\hline \hline 16 & $\begin{array}{l}\text { Zila Sahkari Bank, } \\
\text { Meerut }\end{array}$ & 16 & $\begin{array}{l}\text { Zila Sahkari Bank, } \\
\text { Western Road Branch, } \\
\text { Meerut }\end{array}$ \\
\hline
\end{tabular}

The total number of bank branches selected by the researcher is 16. The customers of these bank branches are chosen for survey using Non-Probability Convenience Sampling.

\section{Sample size for Bank Customers- 320}

\section{(20 Customers per Branch)}

Data Collection: Theresearcher surveys the conveniently available customers of the selected 16 scheduled banks branches of Meerut city to collect from primary data. The respondents are asked to complete the questionnaire by verbally responding to questions in the presence of the researcher.

\section{ANALYSIS AND FINDINGS}

The assessment of concerns, opinions and perceptions of customers, regarding implementation of biometrics in ebanking to find the significant metrics for deployment of biometrics in e-banking with customers perspective is done by analyzing the survey data of customers ( 20 customer per selected bank branch) of selected 16 scheduled banks of Meerut. The closed ended questions in the questionnaire are analyzed using IBM SPSS Statistics 21Statistical Analysis Tool and the few open ended questions in the questionnaires are analyzed using Thematic Textual Analysis.

\subsection{Socio Demographic Information:}

The graph 1.00 shows the bank category wise distribution of respondents (Bank Customers).

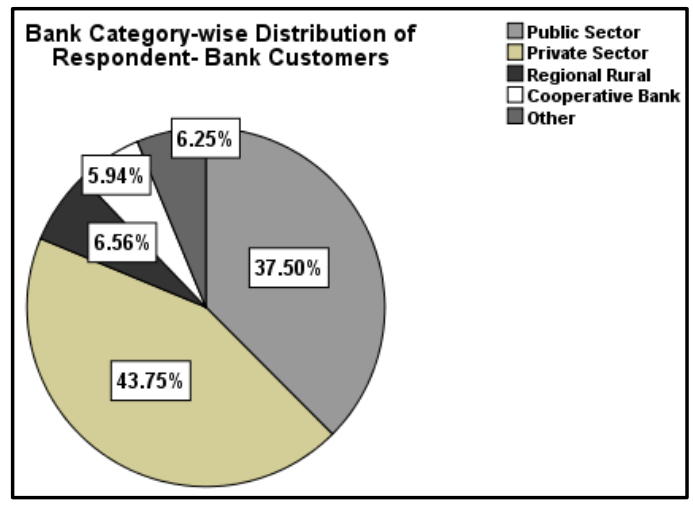

Graph 1.00: Bank Category Wise Distribution of Respondents-Bank Customers

The graph 1.01 shows the gender wise distribution of respondents (Bank Customers). 


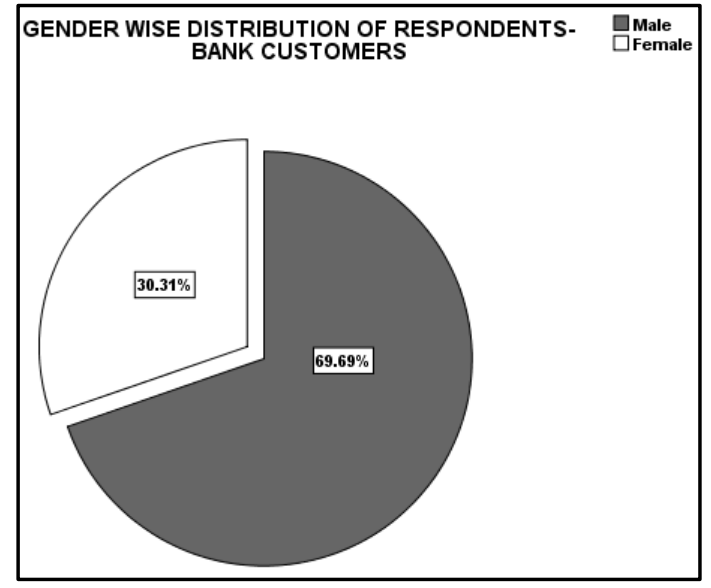

Graph 1.01: Gender wise distribution of respondentsBank customers

The graph 1.02 shows the age group wise distribution of respondents (bank customers).

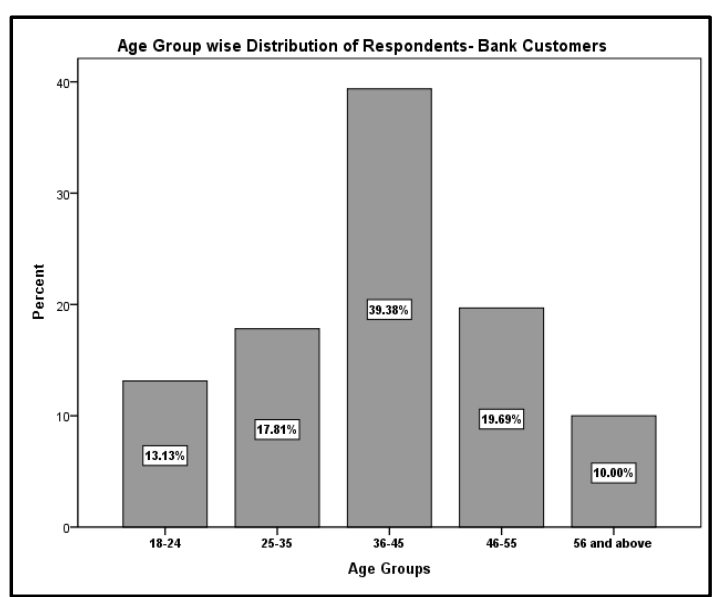

Graph 1.02: Age group wise percentage distribution of respondents- bank customers

The graph 1.03 shows the educational level distribution of respondents (Bank Customers).

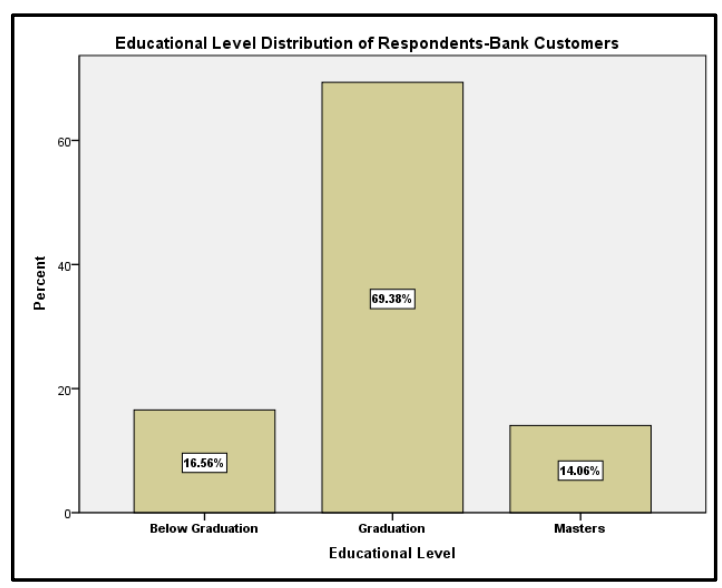

Graph 1.03: Educational level distribution of respondentsBank customers

The graph 1.04 shows the occupation wise distribution of respondents (Bank Customers).

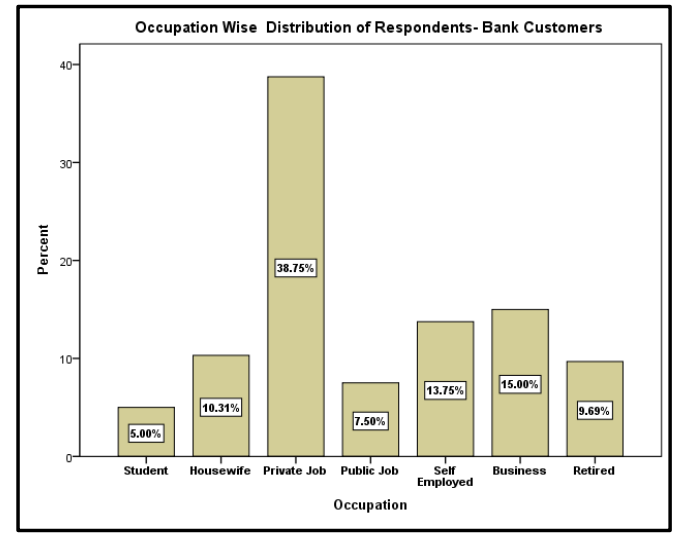

Graph 1.04: Occupation wise distribution of respondentsBank customers

The graph 1.05 shows the Income (Monthly) group wise distribution of respondents (Bank Customers).

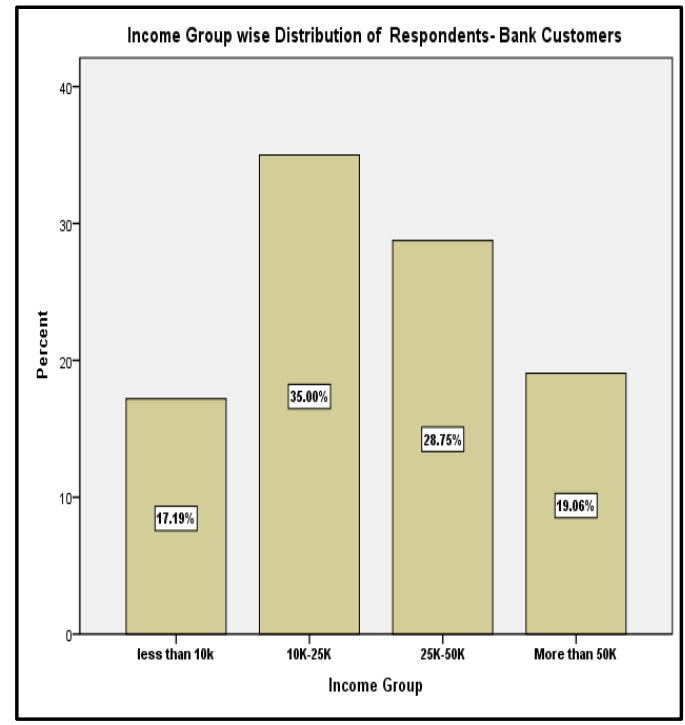

Graph 1.05: Income group wise distribution of respondents- Bank customers

The Graphs 1.00 to 1.05 clearly implies that the selected sample represents a holistic picture of the respondents.

\subsection{Domain Specific Questions}

Responses to question no. 1 of bank customer's questionnaire yields graph 1.06which show the different e-banking channels used by bank customers. From the analysis, it is observed that ATM, Net Banking and Cards are the most preferred channels. 


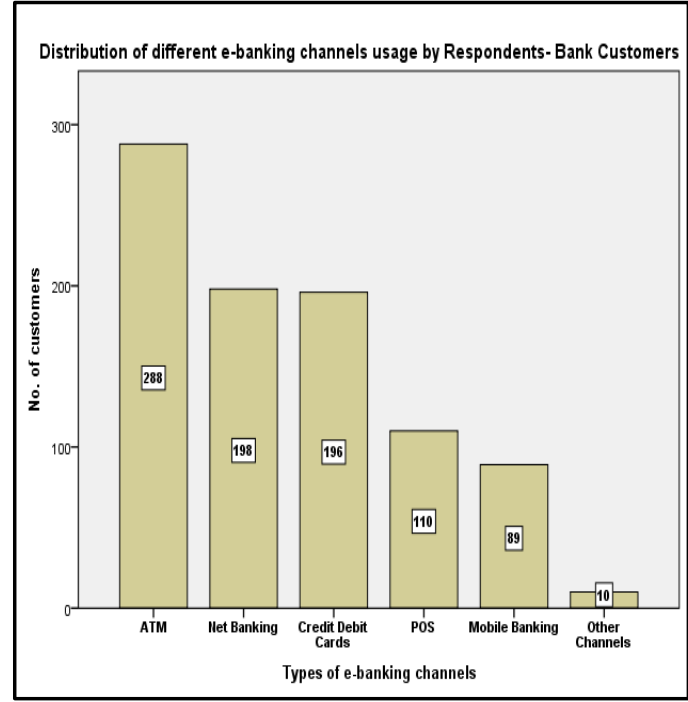

Graph 1.06: Distribution of different e-banking channels usage by bank customers

Responses to question no. 2 of bank customer's questionnaire yields graph 1.07 which show the frequency with which the respondents (Bank Customers) use the e-banking channels. From the analysis, it is observed that almost $84 \%$ respondents use e-banking channels 5 or more times a month, out of which $50 \%$ customers use e-banking channels 5 to 10 times a month and rest more than 10 times a month.

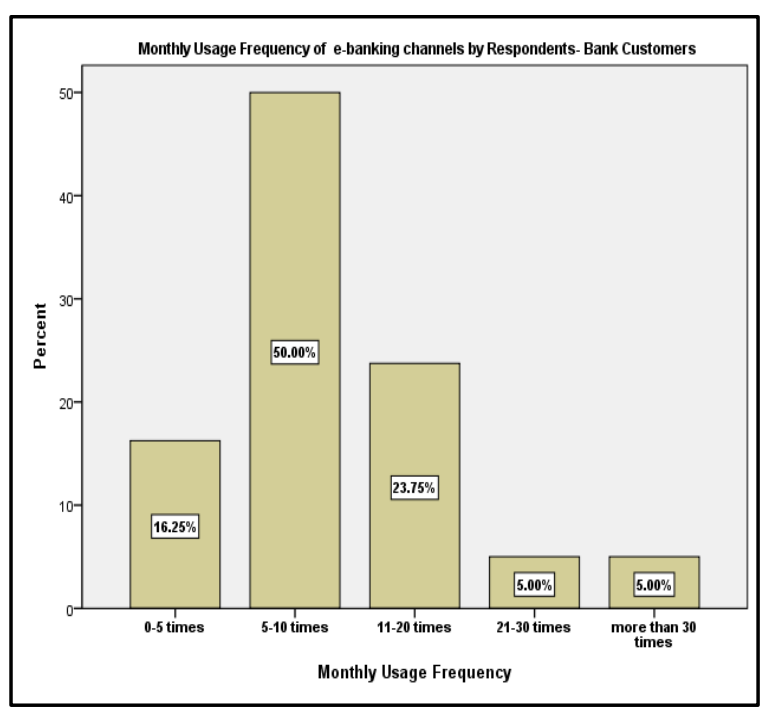

Graph 1.07: Monthly usage frequency of e-banking channels by bank customers

Responses to question no. 3 of bank customers questionnaire yields graph1.08which shows the respondents (Bank Customers)opinion about their concerns of having their online banking account or ATM or Plastic cards (Debit/ Credit / Prepaid Cards etc) being misused or compromised. From the analysis, it is observed that almost $66 \%$ of the customers are concerned about their banking account or ATM or Plastic cards being misused.

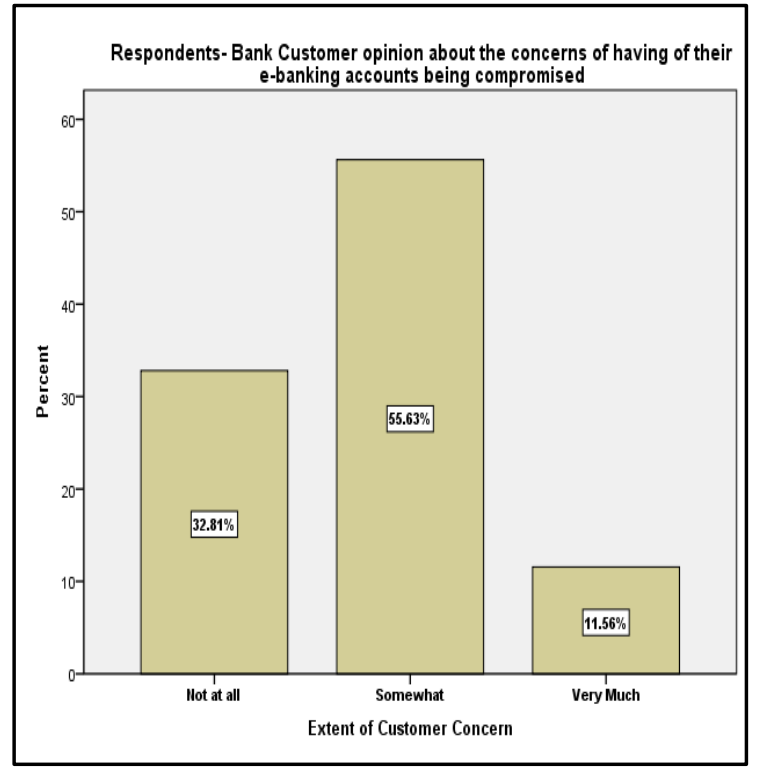

Graph 1.08: Bank customer opinion about the concerns of having of their e-banking accounts being compromised

Responses to question no. 4 of bank customers questionnaire yields graph1.09which shows the whether the respondents (Bank Customers) have ever received a fake email / phone call / message or or having confronted a probable e-banking fraud. From the analysis, it is observed that almost $47.5 \%$ respondents have received a fake email / phone call / message or having confronted a probable e-banking fraud.

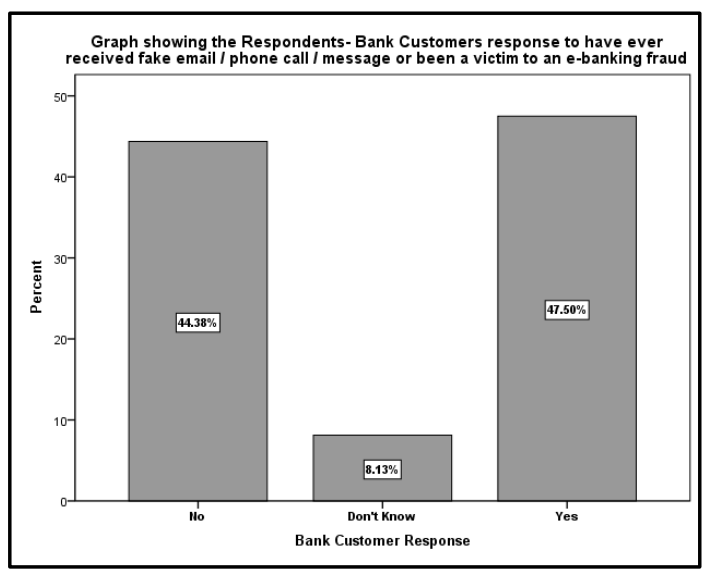

Graph 1.09: Bank customer's response to have ever received fake email / phone call / message or been a victim to an e-banking fraud

Responses to question no. 5 of bank customer's questionnaire yields graph1.10which show the respondents (Bank Customers)opinion about the requirement of additional authentication or verification in e-banking. From the analysis, it is observed that almost $72.5 \%$ of the customers feel that there is a requirement of additional authentication in ebanking. 


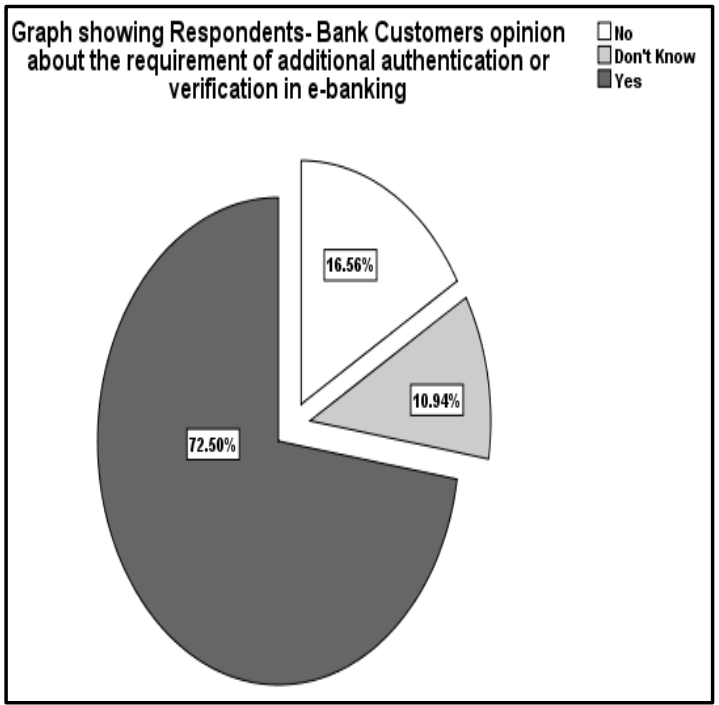

Graph 1.10: Bank customer's opinion about the requirement of additional authentication or verification in e-banking

Among the customers who feel that there is a requirement of additional authentication or verification in e-banking, the responses to the second part of question no. 5 of bank customers questionnaire yields graph1.11 which shows the same respondents (Bank Customers)opinion about the stage the additional authentication or verification should be provided. From the analysis, it is observed that almost $64 \%$ of respondents feel that additional authentication is required on every transaction after login.

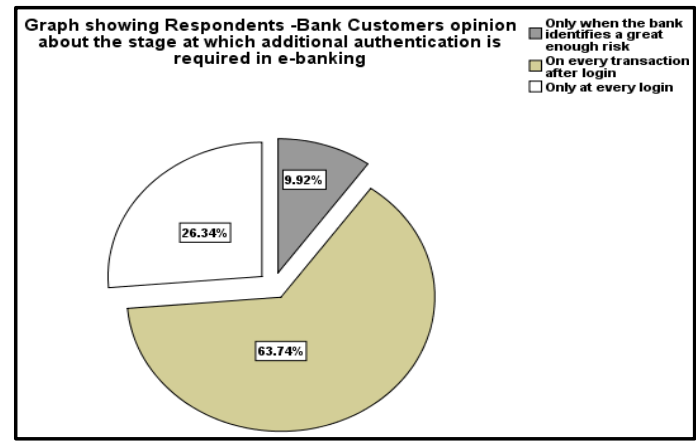

Graph 1.11: Bank customer's opinion about the stage at which additional authentication is required in e-banking

Responses to question no. 6 of bank customer's questionnaire yields graph 1.12 which show the number of different PINs, Passwords, Cards managed by the respondents (Bank Customers). From the analysis, it is observed that almost $47 \%$ respondents manage 5 to 10 and overall $66 \%$ manage more than 5different PINs, Passwords, and Cards.

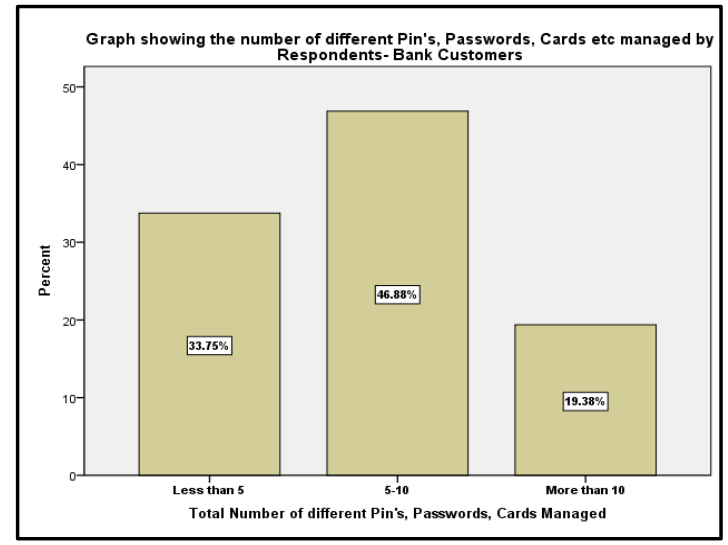

Graph 1.12: Number of different PIN's, passwords, cards etc managed by bank customers

Responses to question no. 7 of bank customers questionnaire yields graph1.13which shows the respondents (Bank Customers)opinion about the problem(s) in managing PIN's, Passwords, Security Questions and Plastic Cards. From the analysis, it is observed that almost $79 \%$ of the customers feel that there are problem(s) in managing PIN's, Passwords, Security Questions and Plastic Cards.

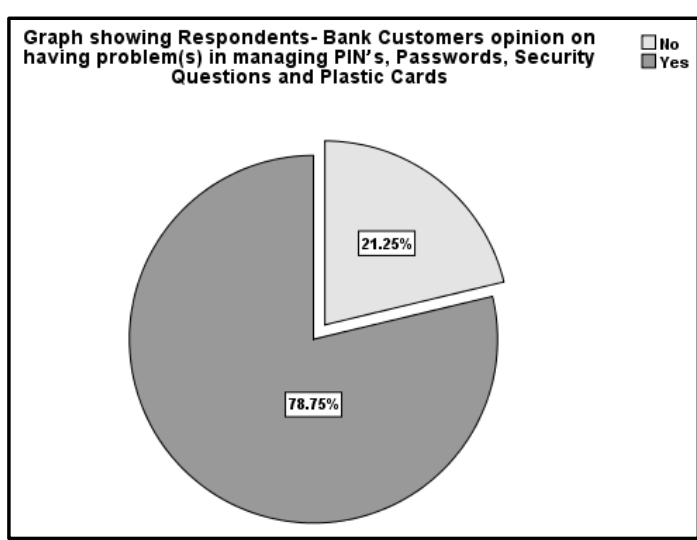

Graph 1.13: Bank customer's opinion on having problem(s) in managing PIN's, passwords, security questions and plastic cards

Among the bank customers who feel that there are problem(s) in managing PIN's, Passwords, Security Questions and Plastic Cards; the responses to the second part of question no. 7 of bank customers questionnaire yields graphs1.14, 1.15, 1.16, $1.17,1.18$ which show the ranking of problem(s) in managing PIN's, Passwords, Security Questions and Plastic Cards by the same respondent's based on the degree of your concern in handling them.

The graph 1.14 shows the bank customers ranking of problems with remembering PIN's, Passwords, and Security Questions. 


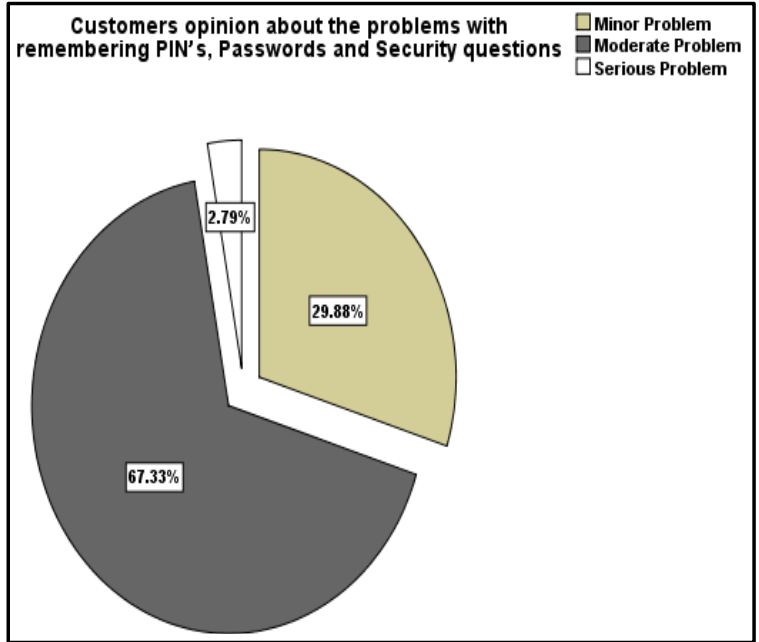

Graph 1.14: Bank managers opinion about the customers of their bank face problems in managing PIN's, passwords etc.

The graph 1.15 shows the bank customers ranking of problems with changing PIN's, Passwords, and Security Questions.

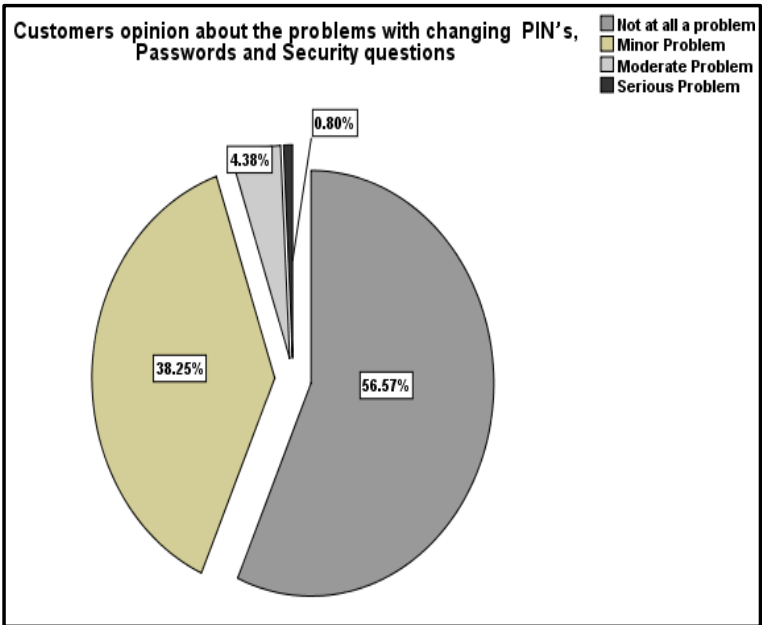

Graph 1.15: Bank customers ranking of problems with changing PIN's, passwords, and security questions

The graph 1.16 shows the bank customers ranking of problems with sharing PIN's, Passwords, and Plastic cards.

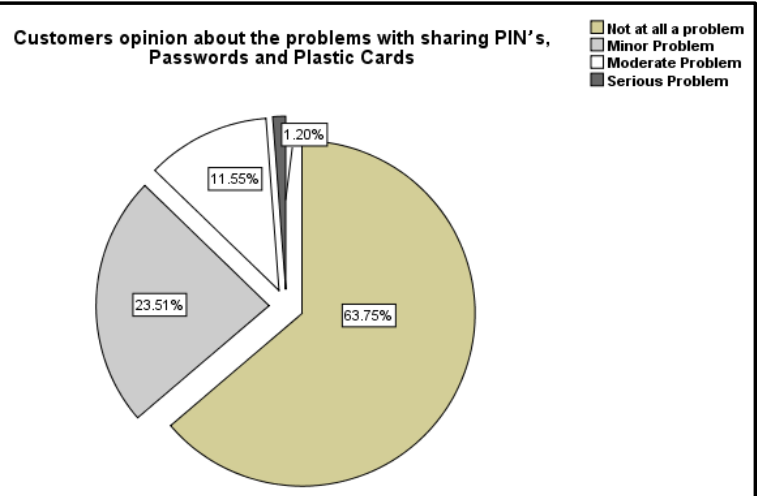

Graph 1.16: Bank customers ranking of problems with sharing PIN's, passwords, and plastic cards
The graph 1.17 shows the bank customers ranking of problems with losing Plastic Cards, PIN's, Passwords or getting stolen.

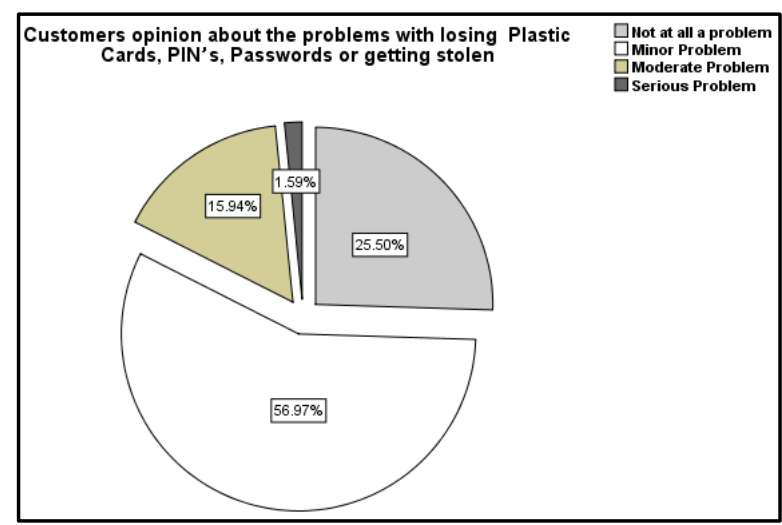

Graph 1.17: Bank customers ranking of problems with losing plastic cards, PIN's, passwords or getting stolen

The graph 1.18 shows the bank customers ranking of any other problems with managing PIN's, Passwords, Security Questions and Plastic Cards.

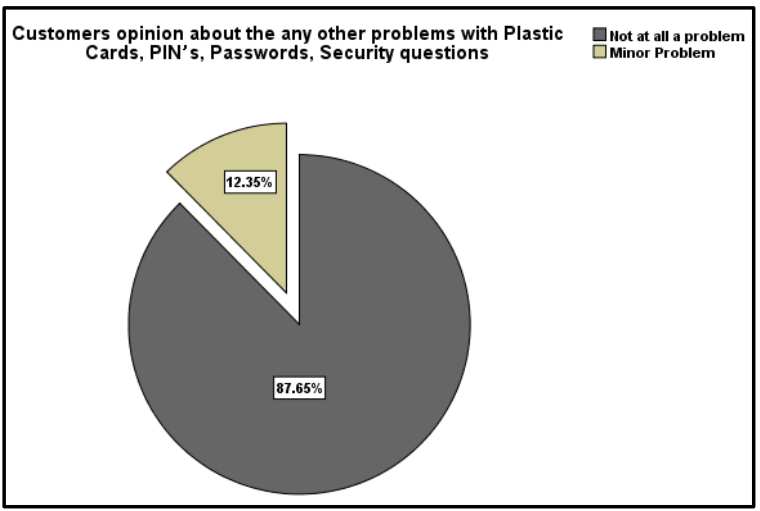

Graph 1.18: Bank customers ranking of any other problems with managing PIN's, passwords, security questions and plastic cards

Responses to question no. 8 of bank customer's questionnaire yields graph1.19which shows that the respondents (Bank Customers)have had personal experience with which biometric technologies. From the analysis, it is observed that most of the respondents have had experience with some biometric technology or other and almost all the respondents have had experience with face and retina recognition, which is mainly due to the aadhaar card programme by the government of India. 


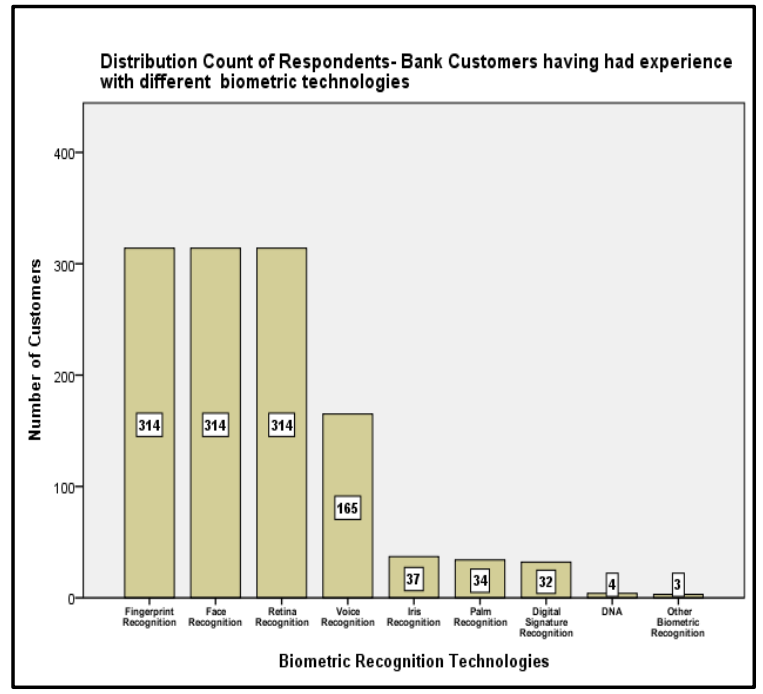

Graph 1.19: Distribution count of bank customers having had experience with different biometric technologies

The responses to the second part of question no. 8 of bank customer's questionnaire yields graph1.20which shows the rating by respondents (Bank Customers)of their experience of using biometric technology. From the analysis, it is observed that almost 59\% respondents have has a good first experience with biometric technology and almost $80 \%$ of the respondents who had average or above average experience.

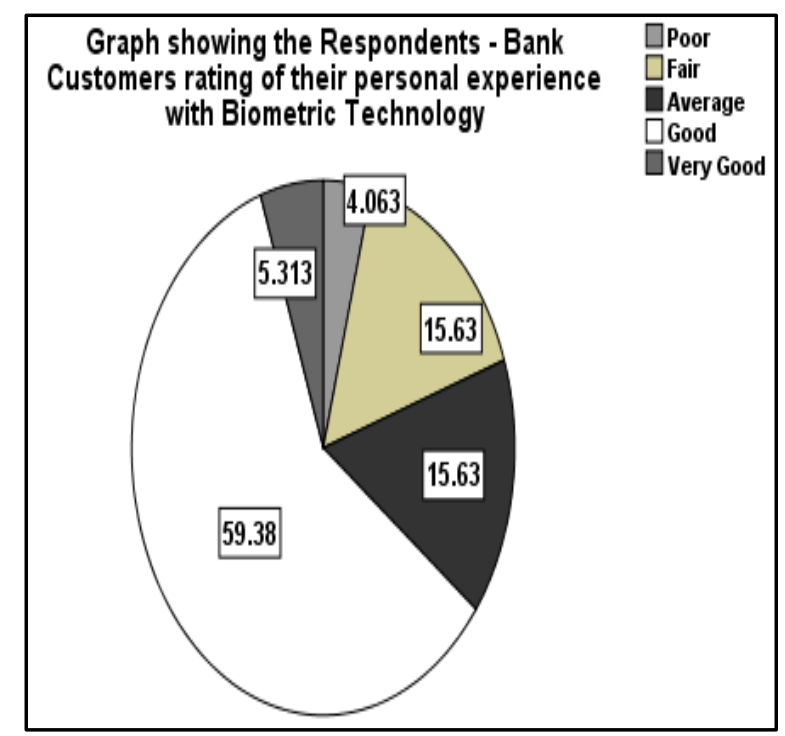

Graph 1.20: Bank customers rating of their personal experience with biometric technology

Responses to question no. 9 of bank customers questionnaire yields graph1.21which shows that the $77 \%$ of the respondents (Bank Customers) agree that biometric authentication will be a good alternative for PIN's, Passwords, Security Questions and Plastic Cards (ATM / Debit/ Credit / Prepaid Cards etc) in e-banking.

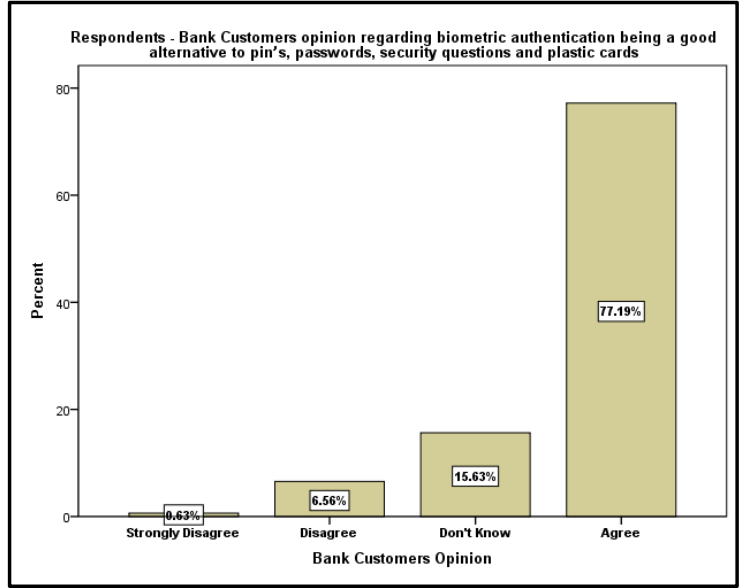

Graph 1.21: Bank customers opinion regarding biometric authentication being a good alternative to pin's, passwords, security questions and plastic cards

Responses to question no. 10 of bank customer's questionnaire yields graph1.22which show that the $78 \%$ of the respondents (Bank Customers) trust biometric technology for secure authentication in e-banking.

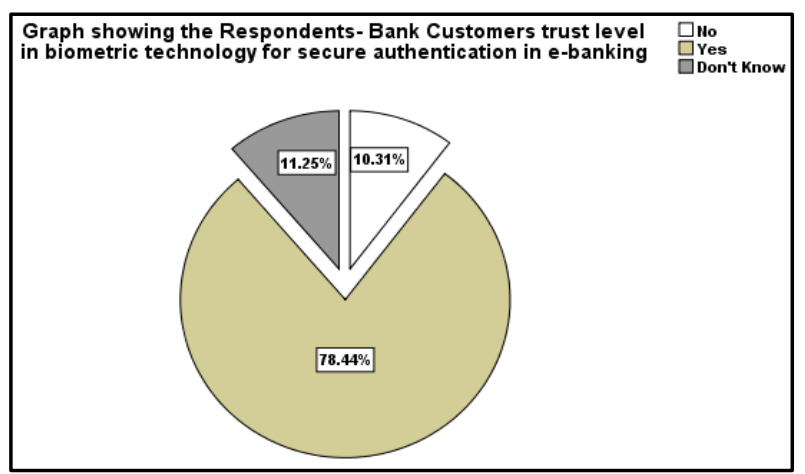

Graph 1.22: Bank customers trust level in biometric technology for secure authentication in e-banking

Responses to question no. 11 of bank customers questionnaire yields graph 1.23 which shows that the $79 \%$ of the respondents (Bank Customers) agree to provide their biometric characteristics like finger print, face, iris scan etc to their bank forenrolment for biometric based authentication.

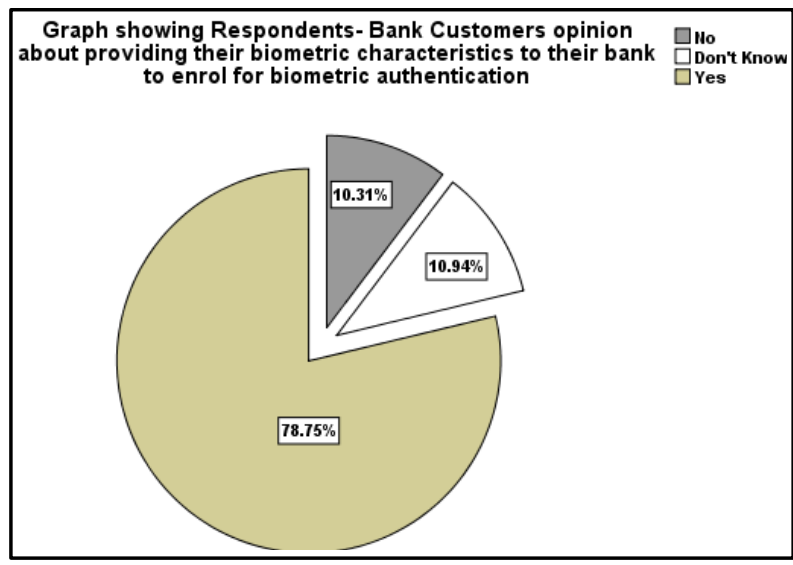

Graph 1.23: Bank customer's opinion about providing their biometric characteristics to their bank to enroll for biometric authentication 
Responses to question no. 12 of bank customers questionnaire yields graph1.24which shows that the $72 \%$ of the respondents (Bank Customers) do not have any concerns about the misuse of your biometric data (fingerprint, face, palm, iris scan etc.) that is taken by banks during enrolment for biometric based authentication.

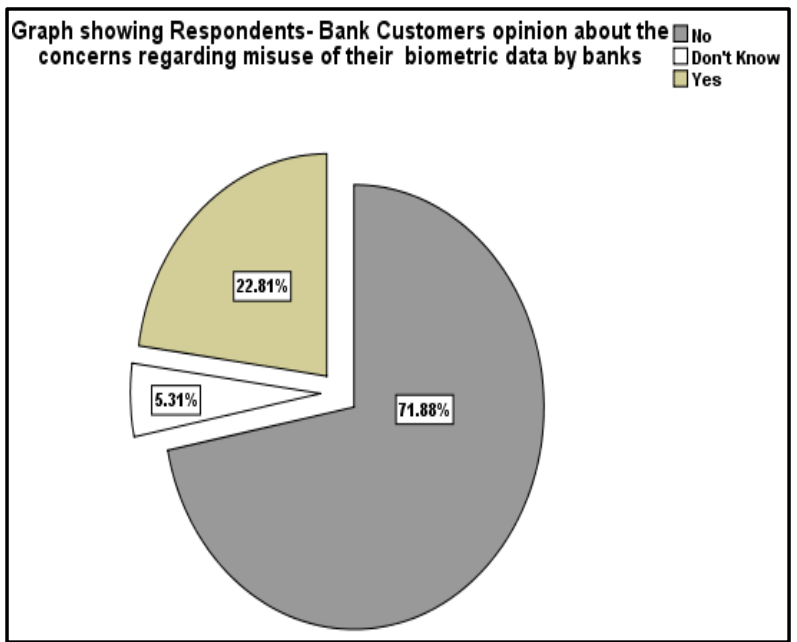

Graph 1.24: Bank customer's opinion about the concerns regarding misuse of their biometric data by banks

Responses to question no. 13 of bank customer's questionnaire yields graph1.25which shows that the $34 \%$ of the respondents (Bank Customers)think biometric technology could be a threat to their privacy.

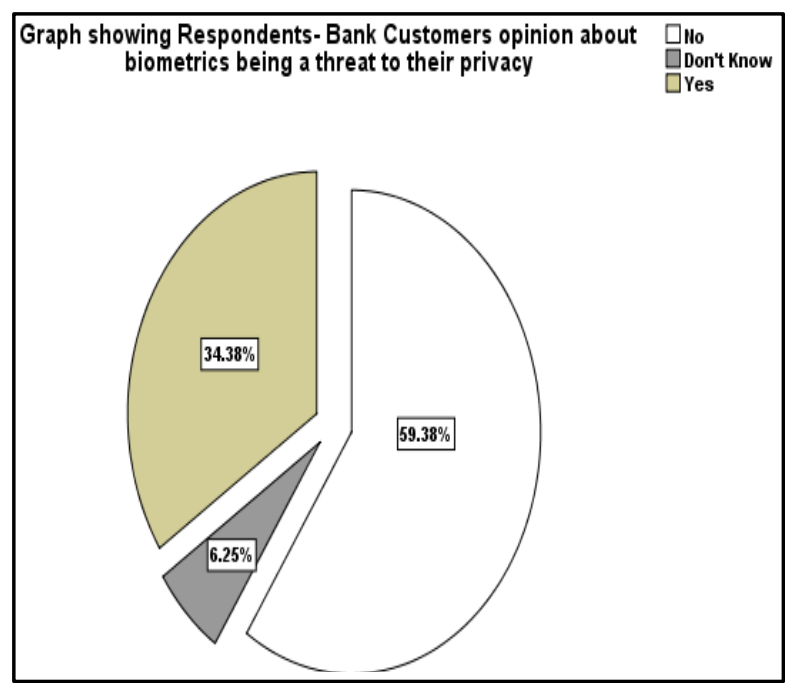

\section{Graph 1.25: Bank customer's opinion about biometrics being a threat to their privacy}

Responses to question no. 14 of bank customer's questionnaire yields graph1.26which show that the $88 \%$ of the respondents (Bank Customers) do not have any health concerns about some biometric technologies that require a physical touch of scanner by different people repeatedly but still a meager $12 \%$ have some health concerns.

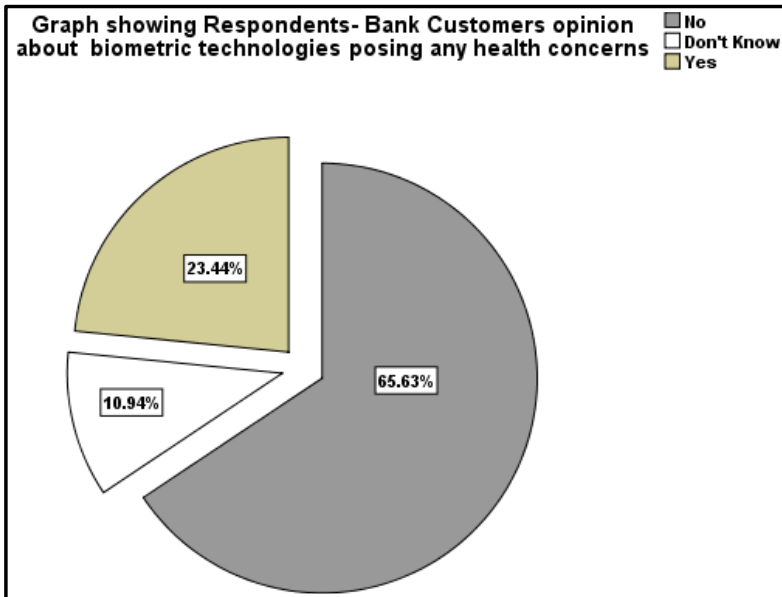

Graph 1.26: Bank customer's opinion about biometric technologies posing any health concerns

The researcher performs thematic textual analysis of the responses to the question no. 15 of bank customer's questionnaire regarding their concerns, opinions and perceptions regarding the deployment of biometric technology in e-banking, this yields the following significant metrics as below:

Table 5.03Significant Metrics regarding the deployment of biometric technology in e-banking as per customers

\begin{tabular}{|c|l|c|}
\hline S. No. & \multicolumn{1}{|c|}{ SIGNIFICANT METRICS } & $\begin{array}{c}\text { Frequency } \\
\text { (n) }\end{array}$ \\
\hline $\mathbf{1}$ & Reliability & $\mathbf{2 3 9}$ \\
\hline $\mathbf{2}$ & Circumvention Resistance & $\mathbf{2 2 1}$ \\
\hline $\mathbf{3}$ & Ergonomics & $\mathbf{1 9 2}$ \\
\hline $\mathbf{4}$ & $\begin{array}{l}\text { Minimum User Participation } \\
\text { (Easy) }\end{array}$ & $\mathbf{9 8}$ \\
\hline $\mathbf{5}$ & Ergonomics (User Friendly) & $\mathbf{7 6}$ \\
\hline
\end{tabular}

Finding Significant Metrics (as per customer's perspective):

From the analysis of question no. 3 to 9 of banker's questionnaire it is observed that reliability and circumvention resistance cost are important metrics in deployment of Biometric technology in E-Banking.

From the analysis of question no. 10, 11 and 12 of banker's questionnaire it is observed that reliability, data security and trust are important metrics indeployment of Biometric technology in E-Banking.

From the analysis of question no. 13 of bank customer's questionnaire it is observed that privacy is an important metric indeployment of Biometric technology in E-Banking.

From the analysis of question no. 14 of bank customer's questionnaire it is observed that health concerns is an important metric indeployment of Biometric technology in EBanking.

From the analysis of question no. 15 of bank customer's questionnaire it is observed that Reliability, Performance, CircumventionResistance, Ergonomics and Minimum User Participation are important metrics indeployment of Biometric technology in E-Banking.

The significant metrics for the deployment of Biometric technology in E-Banking with the bank customer's perspective are: 
- RELIABILITY

- PERFORMANCE

- CIRCUMVENTION RESISTANCE

- ERGONOMICS

- MINIMUM USER PARTICIPATION

- $\quad$ PRIVACY ISSUES

- HEALTH CONCERNS

- DATA SECURITY

\section{- TRUST}

\section{ACKNOWLEDGEMENT}

Author thanks and acknowledges the review assistance of Prof. Anoop Swarup (VC- Jagran Lake City University, Bhopal, INDIA) and Dr. Sohan Garg (Director- Sir Chotu Ram Institute of Engineering and Technology, C.C.S. University, Meerut, U.P. INDIA).

\section{APPENDIX}

The copy of the Sample Survey Questionnaire for Bank Customer's and a file containing all the Graphs, figures and tables used the paper as an addendum.

\section{REFERENCES}

[1] Stanley, P., Jeberson, W., \& Klinsega, V.V, "International Conference on Biometric Authentication: A Trustworthy Technology for Improved Authentication, in Future Network", 2009, pp. 171 175

[2] Russell Kay, "Quick Study: Biometric Authentication", 2009 Accessed: Nov 11, 2014], Available at: <http://www.computerworld.com/s/article/100772/Biom etric_Authentication>

[3] Boatwright, M, \& Luo, X., "What do we know about biometrics authentication?, In Proceedings of the 4th Annual Conference on information Security Curriculum Development", InfoSecCD '07, 2007ACM, New York, pp. 1-5.

[4] Dwivedi, A., Bali, R.K., Belsis, M.A., Naguib, R.N.G., Every, P., \& Nassar, N.S., "4th International IEEE EMBS Special Topic Conference on Towards a practical healthcare information security model for healthcare institutions, Information Technology Applications in Biomedicine", 2003 pp. 114-117.

[5] Chandra, A, \& Calderon, T., "Challenges and constraints to the diffusion of biometrics in information systems", Communication of ACM (ISSN.0001-0782), Vol. 48, No. 12, 2005 pp. 101-106.

[6] Woodward, J.D., 1997, "Biometrics: Privacy's foe or Privacy's friend", Proceedings of the IEEE, Vol.85, No.9, pp. 1480-1492

[7] Wayne Penny, "Biometrics: A Double Edged Sword Security and Privacy", SANS Institute, 2002

[8] Salil Prabhakar, Sharath Pankanti and Anil K. Jain, "Biometric Recognition: Security and Privacy Concerns", THE IEEE COMPUTER SOCIETY, 15407993, 2003 pp.33-42

[9] Chandra, A, \& Calderon, T., 2005, "Challenges and constraints to the diffusion of biometrics in information systems", Communication of ACM (ISSN · 0001-0782), Vol. 48, No.12, pp. 101-106

[10] Elliott, S.J., Massie, S.A., \& Sutton, M.J., "The Perception of Biometric Technology: A Survey,
Automatic Identification Advanced Technologies", IEEE Workshop 2007vol., no., pp. 259-264.

[11] Furnell, S.; and Evangelatos, K., "Public awareness and perceptions of biometrics", Computer Fraud \& Security (ISSN: 1361-3723 Elsevier) Vol. 2007, No. 1, 2007 pp. 8-13.

[12] Andrew Patrick, "Biometrics and Identity Theft", 2007 posted

$<$ http://www.andrewpatrick.ca/essays/biometrics-andidentity-theft/>

[13] Michael Breward, PhD Thesis, "Factors Influencing Consumer Attitudes Towards Iometric Identity Authentication Technology Within The Canadian Banking Industry", School of Graduate Studies, McMaster University

[14] Antonio Pooe and Les Labuschagne, "Investigating The Factors Impacting The Adoption Of Biometric Technology By South African Banks", University of Johannesburg, 2010

[15] A. Pooe (PhD student at the University of South Africa) \& L. Labuschagne (Director of the School of Computing, University of South Africa), "Factors impacting on the adoption of biometric technology by South African banks: An empirical investigation", Southern African Business Review (ISSN: 1998-8125) ,Volume 15 Number 12011 pp. 119

[16] Seyyede Samine Hosseini and Dr. Shahriar Mohammadi, "Acceptance of Banking on Biometric in Iran's Banking SystemCase Study of Saman Bank", , Journal of Basic and Applied Scientific Research, ISSN 2090-4304, 2(12), 2012 pp. 12744-12751

[17] Dhurgham T. Ahmad and Mohammad Hariri, "User Acceptance of Biometrics in E-banking to improve Security", Business Management DynamicsISSN: 2047 7031, Vol.2, No.1,Jul2012, pp.01-04

[18] Sookeun Byun , Sang-Eun Byun, "Exploring perceptions toward biometric technology in service encounters: a comparison of current users and potential adopters", Behaviour \& Information Technology, Vol. 32, Iss. 3, 2013

[19] Adewale Adeyinka A., Ibidunni Ayodotun S., Badejo Joke, Odu Tiwalade and Adoghe Anthony U. , "Biometric Enabled E-Banking in Nigeria: Management and Customers' Perspectives", Information and Knowledge Management” ,ISSN 2224-5758 (Paper) ISSN 2224-896X (Online) Vol.4, No.11, 2014 pp. 23-38

[20] Reserve Bank of India, "A Profile of Banks 2010-11", Mumbai: Director, Division of Reports and Knowledge Dissemination, Department of Economic and Policy Research, Reserve Bank of India (2011) pp 5-89

[21] Reserve Bank of India, "A Profile of Banks 2010-11", Mumbai :Director, Division of Reports and Knowledge Dissemination, Department of Economic and Policy Research, Reserve Bank of India (2011) pp 5-89

\section{AUTHOR PROFILE}

Dr. Munish Sabharwal received his $\mathrm{PhD}$ (Management) from Shobhit University, India in 2013. He is a PGDM (International Trade) from Symbiosis Institute of Management Studies, Pune, India in 2003 and an M.Tech (CSE) from IASE University, Sardarshahr, India in 2005 as well as B.E. (CT) from Nagpur University, Nagpur, India in 1998. He is MCSD and MCP Certified. His current research interests include E- 
Banking, Biometric Technology and Decision Sciences. Specifically, he is interested in designing the framework for authentication and transaction operation for E-Banking using multimodal biometric technology.

He hasover17+ years in Teaching, Education Management, Academic Research as well as Software Developmentand Analysis and is currently spearheading efforts as Executive Director and Professor in KITE Group of Institutions, Meerut (U.P.) INDIA. He has published one book, one E-book and more than 20 academic papers in refereed journals and conferences.

He is Life Member for IAENG (International Association of Engineers, Hong Kong), AIMA (All India Management Association, New Delhi), TAEI (The Association of Engineers India, Kolkata) and YHAI (Youth Hostel Association of India, New Delhi). 